\title{
ON SUMMATION OF CERTAIN DINI SERIES
}

1. Introduction. One of the basic problems that appear when the problems of the mechanics of continuous media (and also others) are dealt with is a physical interpretation of the results obtained. This is especially difficult when the solutions themselves take the form of multiple integrals or series. In particular, when dealing with the problem of heat or mass transport one can often obtain the solutions in a form of FourierBessel or Dini [8] series. The type of the series obtained depends on the kind of boundary conditions. Boundary conditions of the first kind lead frequently to the solution in a form of Fourier-Bessel series whereas boundary conditions of the second or third kind allow us to get the solution often in a form of Dini series. Such series can also appear in other problems, if the region under consideration has a rotational symmetry, the differential equations of the problem are linear and of the second order, and the boundary conditions are expressable as linear combination of the function and its first derivative with respect to the radial variable. Such series also arise when solving the dual series equations [6].

The object of our paper is to sum certain Dini series of the type

$$
\sum_{j=1}^{\infty} \frac{\lambda_{n j}^{2+s} J_{n+k}\left(\lambda_{n j} x\right) J_{n+l}\left(\lambda_{n j} y\right)}{\left(\lambda_{n j}^{2} \pm a^{2}\right)\left(\lambda_{n j}^{2}-n^{2}+H^{2}\right) J_{n}^{2}\left(\lambda_{n j}\right)}
$$

where $J_{n}(z)$ denotes the Bessel function of the first kind and order $n$ (see [3] and [8]), $n=0,1, \ldots, s, k, l=0,1, \lambda_{n s}$ is the $j$-th positive root of the equation $\lambda J_{n}^{\prime}(\lambda)+H J_{n}(\lambda)=0(j=1,2), H$ and $a$ are arbitrary real constants $(a \neq 0)$, and $x, y \in(0,1)$.

The manner of deriving the sums of the series considered rests on the idea of Woelke [9] which is generalized in [1]. That method relies on solving certain partial differential equation in the distributional domain and getting a solution that appears to be certain regular distribution which is the sum of certain series of type (1.1). The equation itself can be created 
by making use of the Lommel integral

$$
\int_{x_{2}}^{x_{1}} x U_{n}(\mu x) V_{n}(\lambda x) d x=\frac{1}{\mu^{2}-\lambda^{2}}\left[\lambda x U_{n}(\mu x) V_{n}^{\prime}(\lambda x)-\mu x V_{n}(\lambda x) U_{n}^{\prime}(\mu x)\right]_{x_{2}}^{x_{1}}
$$

and of the integral

$$
\int_{0}^{z} \xi^{n} U_{n-1}(\xi) d \xi=z^{n} U_{n}(z)
$$

where $\lambda \neq \mu, \lambda$ and $\mu$ are arbitrary real or complex constants being different from zero, $x_{1}>x_{2} \geqslant 0, U_{n}(x)$ and $V_{n}(x)$ are the solutions of the Bessel equation, and $f^{\prime}(z) \equiv d f / d z$.

The basic problem for the summation of the series under consideration is a construction of a sequence of functions $f_{n} \in L^{\text {loc }}(0,1)$ which is of type $\delta$ (see [4] and [7]). In [9] that problem is not treated at all. The author displays only the partial differential equation, the solution of which is used to derive the further formulae. The present paper contains the construction of the sequences of type $\delta$ that are needed to build up the equation which "solves" the problem of summability of the series under consideration.

2. Fundamental series. Let us consider the sequence of functions $\left\{\sigma_{(n) m}(x, y ; H)\right\}_{m=1,2, \ldots}$ that is defined as

$$
\sigma_{(n) m}(x, y ; H)=T_{(n) r}(x, y)-L_{(n) m}(x, y ; H),
$$

where

$$
\begin{aligned}
& T_{(n) r}(x, y)= \begin{cases}2 x \sum_{i=1}^{r} \frac{J_{n}\left(\mu_{n i} x\right) J_{n}\left(\mu_{n i} y\right)}{J_{n+1}^{2}\left(\mu_{n i}\right)} & \text { if } x \in(0,1) \\
0 & \text { if } x \notin(0,1)\end{cases} \\
& L_{(n) m}(x, y ; H)= \begin{cases}2 x\left[\sum_{i=1}^{r} \frac{J_{n}\left(\mu_{n i} x\right) J_{n}\left(\mu_{n i} y\right)}{J_{n+1}^{2}\left(\mu_{n i}\right)}-A_{(n) 0}(x, y)-\right. \\
\left.-\sum_{j=1}^{m} \frac{\lambda_{n j}^{2} J_{n}\left(\lambda_{n j} x\right) J_{n}\left(\lambda_{n j} y\right)}{\left(\lambda_{n j}^{2}-n^{2}+H^{2}\right) J_{n}^{2}\left(\lambda_{n j}\right)}\right] & \text { if } x \in(0,1), \\
0 & \text { if } x \notin(0,1),\end{cases} \\
& A_{(n) 0}(x, y)= \begin{cases}0 & \text { if } H+n>0, \\
(n+1) x^{n} y^{n} & \text { if } H+n=0, \\
\frac{\lambda_{n 0}^{2} I_{n}\left(\lambda_{n 0} x\right) I_{n}\left(\lambda_{n 0} y\right)}{\left(\lambda_{n 0}^{2}+n^{2}+H^{2}\right) I_{n}^{2}\left(\lambda_{n 0}\right)} & \text { if } H+n<0 .\end{cases}
\end{aligned}
$$


Here $\mu_{n i}$ is the $i$-th positive root of the equation $J_{n}(\mu)=0$, the numbers $r$ and $m$ are so chosen that the $r$-th zero $\mu_{n r}$ of the function $J_{n}(\mu)$ lies between $\lambda_{n m}$ and $\lambda_{n, m+1}, \lambda_{n 0}$ stands for the absolute value of the pure imaginary root of the equation $\lambda J_{n}^{\prime}(\lambda)+H J_{n}(\lambda)=0$ if $\boldsymbol{H}+n<0$ [8], and $I_{n}(z)$ denotes the modified Bessel function of the first kind and order $n$ (see [3] and [8]).

The shape of the function $\sigma_{(n) m}(x, y ; H)$ is chosen in the above-described way because of the properties of the sequences $\left\{T_{(n) r}(x, y)\right\}_{r=1,2, \ldots}$ and $\left\{L_{(n) m}(x, y ; H)\right\}_{m=1,2, \ldots}$. The following theorems hold:

THEOREM 1 (Watson [8]). If $(a, b)$ is any part of the closed interval $[0,1]$ such that $y$ is not an internal point or an end point of $(a, b)$, then the existence and the absolute convergence of $\int_{a}^{b} \sqrt{t} f(t) d t$ are sufficient to ensure

$$
\lim _{n \rightarrow \infty} \int_{a}^{b} T_{(n) r}(x, y) f(x) d x=0
$$

where $0<y \leqslant 1$. (If $y=1$, it is, of course, supposed that $b<1$.)

Theorem 2 (Watson [8]). Let $f(x)$ be a function defined arbitrarily in the interval $(0,1)$ and assume that $\int_{0}^{1} \sqrt{x} f(x) d x$ exists and (if it is an improper integral) is absolutely convergent. Let

$$
\dot{a}_{(n) i}=\frac{2}{J_{n+1}^{2}\left(\mu_{n i}\right)} \int_{0}^{1} x f(x) J_{n}\left(\mu_{n i} x\right) d x,
$$

where $n=0,1, \ldots$ Let $y$ be any internal point of an interval $(a, b)$ such that $0<a<b<1$ and such that $f(x)$ satisfies the Dirichlet conditions [5] in $(a, b)$. Then the series

$$
\sum_{i=1}^{\infty} a_{(n) i} J_{n}\left(\mu_{n i} y\right)
$$

is convergent and its sum is $\frac{1}{2}\{f(y+0)+f(y-0)\}$.

Corollary 1. If $y \in(a, b) \subset(0,1)$, then

$$
\lim _{r \rightarrow \infty} \int_{a}^{b} T_{(n) r}(x, y) d x=1 .
$$

Theorem 3 (Szmydt [7]). Let $f_{r} \in L^{\text {loc }}\left(E^{1}\right)(r=1,2, \ldots)$ and suppose that

(i) for every $M>0$ there exists a constant $C_{1}<+\infty$ such that

$$
\left|\int_{t_{1}}^{t_{2}} f_{r}(t) d t\right|<C \text { for }\left|t_{1}\right|,\left|t_{2}\right|<M ;
$$


(ii) for all $t_{1}, t_{2}$ with $t_{1} \neq 0 \neq t_{2}, t_{1} \leqslant t_{2}$, we have

$$
\lim _{r \rightarrow \infty} \int_{t_{1}}^{t_{2}} f_{r}(t) d t= \begin{cases}0 & \text { if } t_{1} t_{2}>0 \\ 1 & \text { if } t_{1} t_{2}<0\end{cases}
$$

Then the sequence $\left\{f_{r}\right\}$ is of type $\delta$.

CoRollary 2. The sequence $\left\{T_{(n) r}(x, y)\right\}_{r=1,2, \ldots}$ is of type $\delta$ with respect to the variable $x$.

Proof. We must show that the assumptions of Theorem 3 are fulfilled. Taking into account $T_{(n) r}$ as a function of $x$ we can easily prove that it is locally integrable by virtue of (2.2). Assumption (i) is fulfilled by virtue of Theorem 1 , and (ii) is also satisfied because of Theorem 1 and Corollary 1 (it suffices to substitute $x=t+y$ into the theorem and the corollary to get them in the form of the assumptions of Theorem 3). Thus we have proved that the sequence $\left\{T_{(n) r}\right\}$ is of type $\delta$.

Theorem 4 (Watson [8]). If $(a, b)$ is any part (or the whole) of the interval $(0,1)$, then the existence and absolute convergence of $\int_{a}^{b} \sqrt{x} f(x) d x$ are sufficient to ensure that

$$
\lim _{n \rightarrow \infty} \int_{a}^{b} f(x) L_{(n) m}(x, y ; H) d x=0
$$

provided that $y \in(0,1)$. And, if $b<1$, the theorem is valid when $y \in(0,1]$.

THEOREM 5. The sequence $\left\{\sigma_{(n) m}(x, y ; H)\right\}_{n=1,2, \ldots}$, defined by (2.1), when considered as a sequence of functions of the variable $x$, is of type $\delta$.

Proof. It is evident from the definition of $\sigma_{(n) m}$ and from (2.2) and (2.3) that $\sigma_{(n) m}$ is locally integrable. Hence we must prove only that assumptions (i) and (ii) of Theorem 3 are satisfied. By virtue of Theorem 4 we can easily show that

$$
\lim _{m \rightarrow \infty} \int_{t_{1}}^{t_{2}} L_{(n) m}(x, y ; H) d x=0 .
$$

To get (2.5) it suffices to choose a function $f(x)$ as follows:

$$
f(x)= \begin{cases}1 & \text { if } x \in\left(t_{1}, t_{2}\right) \subset(0,1) \\ 0 & \text { if } x \in(0,1) \backslash\left[t_{1}, t_{2}\right]\end{cases}
$$

Hence for every $M>0$ there exists a constant $C_{1}<+\infty$ such that $\left|\int_{t_{1}}^{t_{2}} \sigma_{(n) m}(t, y ; H) d t\right| \leqslant\left|\int_{t_{1}}^{t_{2}} T_{(n) r}(t, y) d t\right|+\left|\int_{t_{1}}^{t_{2}} L_{(n) m}(t, y ; H) d t\right|<C+C_{L}=C_{1}$ for $\left|t_{1}\right|,\left|t_{2}\right|<M$. We have $C_{L}<+\infty$ because of (2.5). Thus assumption (i) 
of Theorem 3 is satisfied. By virtue of (2.5) we also can write the equality

$$
\lim _{m \rightarrow \infty} \int_{t_{1}}^{t_{2}} \sigma_{(n) m}(t, y ; H) d t=\lim _{r \rightarrow \infty} \int_{t_{1}}^{t_{2}} T_{(n) r}(t, y) d t,
$$

which ensures - by virtue of Corollary 2 - the validity of assumption (ii). Thus the sequence $\left\{\sigma_{(n) m}\right\}$ is of type $\delta$.

As a result of Corollary 2 and Theorem 5 we can state that for all $\varphi(x) \in C_{0}^{\infty}$ (see [7]) the following equalities hold:

$$
\begin{gathered}
\lim _{r \rightarrow \infty} \int_{-\infty}^{+\infty} T_{(n) r}(x, y) \varphi(x) d x=\varphi(y)=(\delta(x-y), \varphi(x)), \\
\lim _{m \rightarrow \infty} \int_{-\infty}^{+\infty} \sigma_{(n) m}(x, y ; H) \varphi(x) d x=\varphi(y)=(\delta(x-y), \varphi(x)) .
\end{gathered}
$$

Making use of (2.2) and (2.3) we can rewrite $\sigma_{(n) m}(x, y ; H)$ in the form

$$
\sigma_{(n) m}(x, y ; H)=2 x\left\{A_{(n) 0}(x, y)+\sum_{j=1}^{m} \frac{\lambda_{n j}^{2} J_{n}\left(\lambda_{n j} x\right) J_{n}\left(\lambda_{n j} y\right)}{\left(\lambda_{n j}^{2}-n^{2}+H^{2}\right) J_{n}^{2}\left(\lambda_{n j}\right)}\right\},
$$

where $x, y \in(0,1)$ and $A_{(n) 0}(x, y)$ is the function defined by (2.4).

Thus - by virtue of (2.2), (2.8), (2.6) and (2.7) - we can write the distributional equalities

$$
\begin{gathered}
2 x \sum_{i=1}^{\infty} \frac{J_{n}\left(\mu_{n i} x\right) J_{n}\left(\mu_{n i} y\right)}{J_{n+1}^{2}\left(\mu_{n i}\right)}=\delta(y-x), \\
2 x\left\{A_{(n) 0}(x, y)+\sum_{j=1}^{\infty} \frac{\lambda_{n j}^{2} J_{n}\left(\lambda_{n j} x\right) J_{n}\left(\lambda_{n j} y\right)}{\left(\lambda_{n j}^{2}-n^{2}+H^{2}\right) J_{n}^{2}\left(\lambda_{n j}\right)}\right\}=\delta(y-x),
\end{gathered}
$$

where $n=0,1,2, \ldots$ and $x, y \in(0,1)$.

Let us multiply distributionally the both sides of equalities (2.9) and (2.10) by $I_{n}(a x)[\eta(x)-\eta(x-\bar{x})]$ with $0<y<\bar{x}<1$. Choosing a test function to be a constant for $x \in(0,1)$ and making use of (1.2) and (1.3) we arrive at results which can be written in the form of the following partial differential equations of the first order (in what follows we neglect the overlining over the variable $x$ ):

$$
\left\{\begin{array}{l}
S_{n}(x, y) \\
S_{n}^{H}(x, y)
\end{array}\right\}-\frac{I_{n}(a x)}{a I_{n}^{\prime}(a x)} \frac{\partial}{\partial x}\left\{\begin{array}{l}
S_{n}(x, y) \\
S_{n}^{H}(x, y)
\end{array}\right\}=\frac{I_{n}(a y)}{2 a x I_{n}^{\prime}(a x)}
$$

Here

$$
\begin{aligned}
& S_{n}(x, y)=\sum_{i=1}^{\infty} \frac{J_{n}\left(\mu_{n i} x\right) J_{n}\left(\mu_{n i} y\right)}{\left(\mu_{n i}^{2}+a^{2}\right) J_{n+1}^{2}\left(\mu_{n i}\right)} \\
& S_{n}^{H}(x, y)=\sum_{j=1}^{\infty} \frac{\lambda_{n j}^{2} J_{n}\left(\lambda_{n j} x\right) J_{n}\left(\lambda_{n j} y\right)}{\left(\lambda_{n j}^{2}+a^{2}\right)\left(\lambda_{n j}^{2}-n^{2}+H^{2}\right) J_{n}^{2}\left(\lambda_{n j}\right)}+S_{(n) 0}(x, y ; a)
\end{aligned}
$$


with

$$
S_{(n) 0}(x, y ; a)= \begin{cases}(n+1) x^{n} y^{n} a^{-2} & \text { if } H+n=0, \\ \frac{\eta(H+n)-1}{\lambda_{n 0}^{2}-a^{2}} A_{(n) 0}(x, y) & \text { otherwise, }\end{cases}
$$

$\eta$ being the Heaviside function.

The solution of (2.11) takes the form

$$
B_{n} I_{n}(x)+\frac{1}{2} I_{n}(a y) K_{n}(a x),
$$

where $B_{n}$ is a constant which can be defined by the condition

$$
S_{n}(1, y)=0 \quad \text { when solving }(2.11)_{1}
$$

or

$$
H S_{n}^{H}(1, y)+\left[\frac{\partial}{\partial x} S_{n}^{H}(x, y)\right]_{x=1}=0 \quad \text { when solving (2.11) },
$$

and $K_{n}$ denotes the modified Bessel function of the second kind and order $n$ [8].

Since both $S_{n}$ and $S_{n}^{H}$ are symmetric in $x$ and $y$, taking into consideration (2.13), (2.14) and (2.15) we can obtain the relations

$$
\begin{aligned}
& \sum_{i=1}^{\infty} \frac{J_{n}\left(\mu_{n i} x\right) J_{n}\left(\mu_{n i} y\right)}{\left(\mu_{n i}^{2}+a^{2}\right) J_{n+1}^{2}\left(\mu_{n i}\right)} \\
& =2 a^{2}\left\{\eta(x-y) F_{n 1}(a, x) I_{n}(a y)+\eta(y-x) F_{n 1}(a, y) I_{n}(a x)\right\}
\end{aligned}
$$

The distributions defined by (2.16) and (2.17) are regular and may be treated as functions [7].

We introduced for brevity the following symbols into (2.16) and (2.17):

$$
\begin{aligned}
& F_{n 1}(p, z)=\frac{1}{4 p^{2}}\left[K_{n}(p z)-I_{n}(p z) \frac{K_{n}(p)}{I_{n}(p)}\right] \\
& F_{n 1}^{H}(p, z)=\frac{1}{4 p^{2}}\left[K_{n}(p z)-I_{n}(p z) \frac{(H+n) K_{n}(p)-p K_{n+1}(p)}{(H+n) I_{n}(p)+p I_{n+1}(p)}\right] .
\end{aligned}
$$

In what follows we make also use of the abbreviation

$$
F_{n 2}^{B}(p, z)=\frac{1}{4 p^{2}}\left[K_{n+1}(p z)+I_{n+1}(p z) \frac{(H+n) K_{n}(p)-p K_{n+1}(p)}{(H+n) I_{n}(p)+p I_{n+1}(p)}\right]
$$


Relation (2.16) is the fundamental one for deriving the summation formulae for the Fourier-Bessel scries. In this paper we do not deal with those since they are derived in [1]. In the further analysis we make use of formula (2.17) that is the fundamental one for deriving the summation formulae for the Dini series of type (1.1).

In what follows we often speak about passing to the limit with both sides of the formulae for $x$ or $y$ tending to 1 . The uniformity of convergence of the Dini series in this case is ensured by the considerations and theorems being given in [8], Sections 18.34 and 18.35 .

3. Other series of type (1.1). Let us differentiate distributionally formula (2.17) with respect to $x$. We obtain

$$
\begin{aligned}
& \sum_{j=1}^{\infty} \frac{\lambda_{n j}^{3} J_{n+1}\left(\lambda_{n j} x\right) J_{n}\left(\lambda_{n j} y\right)}{\left(\lambda_{n j}^{2}+a^{2}\right)\left(\lambda_{n j}^{2}-n^{2}+H^{2}\right) J_{n}^{2}\left(\lambda_{n j}\right)} \\
& =\frac{\lambda_{n 0}[\eta(H+n)-1] I_{n+1}\left(\lambda_{n 0} x\right)}{\left(\lambda_{n 0}^{2}-a^{2}\right) I_{n}\left(\lambda_{n 0} x\right)} \bar{A}_{(n) 0}(x, y)+ \\
& +2 a^{3}\left\{\eta(x-y) F_{n 2}^{H}(a, x) I_{n}(a y)-\eta(y-x) F_{n 1}^{H}(a, y) I_{n+1}(a x)\right\}
\end{aligned}
$$

where

$$
\bar{A}_{(n) 0}(x, y)= \begin{cases}A_{(n) 0}(x, y) & \text { if } H+n \neq 0, \\ 0 & \text { if } H+n=0,\end{cases}
$$

the function $A_{(n) 0}(x, y)$ being defined by (2.4).

If $a \rightarrow 0$ in (3.1), we obtain

$$
\begin{aligned}
& \sum_{j=1}^{\infty} \frac{\lambda_{n j} J_{n+1}\left(\lambda_{n j} x\right) J_{n}\left(\lambda_{n j} y\right)}{\left(\lambda_{n j}^{2}-n^{2}+H^{2}\right) J_{n}^{2}\left(\lambda_{n j}\right)} \\
& \quad=[\eta(H+n)-1] \frac{I_{n+1}\left(\lambda_{n 0} x\right)}{\lambda_{n 0} I_{n}\left(\lambda_{n 0} x\right)} \bar{A}_{(n) 0}(x, y)+\frac{\eta(x-y)}{2 x}\left(\frac{y}{x}\right)^{n} .
\end{aligned}
$$

Multiplying both sides of (3.1) by $-a^{-2}$ and both sides of (3.2) by $a^{-2}$ and then adding we get

$$
\begin{gathered}
\sum_{j=1}^{\infty} \frac{\lambda_{n j} J_{n+1}\left(\lambda_{n j} x\right) J_{n}\left(\lambda_{n j} y\right) \cdot}{\left(\lambda_{n j}^{2}+a^{2}\right)\left(\lambda_{n j}^{2}-n^{2}+H^{2}\right) J_{n}^{2}\left(\lambda_{n j}\right)}=\frac{[1-\eta(H+n)] I_{n+1}\left(\lambda_{n 0} x\right)}{\lambda_{n 0}\left(\lambda_{n 0}^{2}-a^{2}\right) I_{n}\left(\lambda_{n 0} x\right)} \bar{A}_{(n) 0}(x, y)+ \\
+\eta(x-y)\left\{\left(2 a^{2} x\right)^{-1}\left(\frac{y}{x}\right)^{n}-2 a F_{n 2}^{H}(a, x) I_{n}(a y)\right\}+ \\
+\eta(y-x) 2 a F_{n 1}^{H}(a, y) I_{n+1}(a x) .
\end{gathered}
$$

Differentiating distributionally (3.3) with respect to $y$ we obtain

$$
\begin{aligned}
& \sum_{j=1}^{\infty} \frac{\lambda_{n j}^{2} J_{n+1}\left(\lambda_{n j} x\right) J_{n+1}\left(\lambda_{n j} y\right)}{\left(\lambda_{n j}^{2}+a^{2}\right)\left(\lambda_{n j}^{2}-n^{2}+H^{2}\right) J_{n}^{2}\left(\lambda_{n j}\right)} \\
& =\frac{[\eta(H+n)-1] I_{n+1}\left(\lambda_{n 0} x\right) I_{n+1}\left(\lambda_{n 0} y\right)}{\left(\lambda_{n 0}^{2}-a^{2}\right) I_{n}\left(\lambda_{n 0} x\right) I_{n}\left(\lambda_{n 0} y\right)} \bar{A}_{(n) 0}+ \\
& +2 a^{2}\left\{\eta(x-y) F_{n 2}^{H}(a, x) I_{n+1}(a y)+\eta(y-x) F_{n 2}^{H}(a, y) I_{n+1}(a x)\right\}
\end{aligned}
$$


Making use of (2.17), (3.1), (3.3) and (3.4) we can derive (for $a \neq b$ ) the summation formulae

$$
\sum_{j=1}^{\infty} \frac{\lambda_{n j}^{2} J_{n}\left(\lambda_{n j} x\right) J_{n}\left(\lambda_{n j} y\right)}{\left(\lambda_{n j}^{2}+a^{2}\right)\left(\lambda_{n j}^{2}+b^{2}\right)\left(\lambda_{n j}^{2}-n^{2}+H^{2}\right) J_{n}^{2}\left(\lambda_{n j}\right)}
$$

$$
\begin{gathered}
=\overline{\bar{A}}_{(n) 0}(x, y)+\frac{2}{b^{2}-a^{2}}\left\{\eta(x-y)\left[a^{2} F_{n 1}^{H}(a, x) I_{n}(a y)-b^{2} F_{n 1}^{H}(b, x) I_{n}(b y)\right]+\right. \\
\left.+\eta(y-x)\left[a^{2} F_{n 1}^{H}(a, y) I_{n}(a x)-b^{2} F_{n 1}^{H}(b, y) I_{n}(b x)\right]\right\},
\end{gathered}
$$

where

$$
\begin{gathered}
\overline{\bar{A}}_{(n) 0}(x, y)=\left\{\begin{array}{l}
\frac{-A_{(n) 0}(x, y)}{\left(\lambda_{n 0}^{2}-a^{2}\right)\left(\lambda_{n 0}^{2}-b^{2}\right)} \quad \text { if } H+n \neq 0, \\
-\frac{(n+1) x^{n} y^{n}}{a^{2} b^{2}} \quad \text { if } H+n=0 ;
\end{array}\right. \\
\sum_{j=1}^{\infty} \frac{\lambda_{n j}^{3} J_{n+1}\left(\lambda_{n j} x\right) J_{n}\left(\lambda_{n j} y\right)}{\left(\lambda_{n j}^{2}+a^{2}\right)\left(\lambda_{n j}^{2}+b^{2}\right)\left(\lambda_{n j}^{2}-n^{2}+H^{2}\right) J_{n}^{2}\left(\lambda_{n j}\right)} \\
=\frac{[1-\eta(H+n)] \lambda_{n 0} I_{n+1}\left(\lambda_{n 0} x\right) \bar{A}_{(n) 0}(x, y)}{\left(\lambda_{n 0}^{2}-a^{2}\right)\left(\lambda_{n 0}^{2}-b^{2}\right) I_{n}\left(\lambda_{n 0} x\right)}+ \\
+\frac{2}{b^{2}-a^{2}}\left\{\eta(x-y)\left[a^{3} F_{n 2}^{H}(a, x) I_{n}(a y)-b^{3} F_{n 2}^{H}(b, x) I_{n}(b y)\right]-\right. \\
\sum_{j=1}^{\infty} \frac{\left.-\eta(y-x)\left[a^{3} F_{n 1}^{H}(a, y) I_{n+1}(a x)-b^{3} F_{n 1}^{H}(b, y) I_{n+1}(b x)\right]\right\}}{\left(\lambda_{n j}^{2}+a^{2}\right)\left(\lambda_{n j}^{2}+b^{2}\right)\left(\lambda_{n j}^{2}-n^{2}+H^{2}\right) J_{n}^{2}\left(\lambda_{n j}\right)} \\
=\frac{\eta(x-y)}{2 a^{2} b^{2} x}\left(\frac{y}{x}\right)^{n}+\frac{[\eta(H+n)-1] I_{n+1}\left(\lambda_{n 0} x\right) \bar{A}_{(n) 0}(x, y)}{\lambda_{n 0}\left(\lambda_{n 0}^{2}-a^{2}\right)\left(\lambda_{n 0}^{2}-b^{2}\right) I_{n}\left(\lambda_{n 0} x\right)}+ \\
+\frac{2}{b^{2}-a^{2}}\left\{\eta(x-y)\left[b F_{n 2}^{H}(b, x) I_{n}(b y)-a F_{n 2}^{H}(a, x) I_{n}(a y)\right]+\right. \\
\left.+\eta(y-x)\left[a F_{n 1}^{H}(a, y) I_{n+1}(a x)-b F_{n 1}^{H}(b, y) I_{n+1}(b x)\right]\right\} \\
+\frac{2}{\left(\lambda_{n 0}^{2}-a^{2}\right)\left(\lambda_{n 0}^{2}-b^{2}\right) I_{n}\left(\lambda_{n 0} x\right) I_{n}\left(\lambda_{n 0} y\right)} \\
\sum_{j=1}^{\infty} \frac{2}{b^{2}-a^{2}}\left\{\eta(x-y)\left[a^{2} F_{n 2}^{H}(a, x) I_{n+1}(a y)-b^{2} F_{n 2}^{H}(b, x) I_{n+1}(b y)\right]+\right. \\
\left.+\eta(y-x)\left[a^{2} F_{n 2}^{H}(a, y) I_{n+1}(a x)-b^{2} F_{n 2}^{H}(b, y) I_{n+1}(b x)\right]\right\}+ \\
+\frac{\left[1-\eta\left(\lambda_{n j}^{2}+b^{2}\right)\left(\lambda_{n j}^{2}-n^{2}+H^{2}\right) J_{n}^{2}\left(\lambda_{n j}\right)\right.}{\left(\lambda_{n+1}\left(\lambda_{n j} y\right)\right.}
\end{gathered}
$$




$$
\sum_{j=1}^{\infty} \frac{J_{n+1}\left(\lambda_{n j} x\right) J_{n+1}\left(\lambda_{n j} y\right)}{\left(\lambda_{n j}^{2}+b^{2}\right)\left(\lambda_{n j}^{2}-n^{2}+H^{2}\right) J_{n}^{2}\left(\lambda_{n j}\right)}
$$$$
=\eta(x-y)\left\{\frac{(y / x)^{n+1}}{4 b^{2}(n+1)}-2 F_{n 2}^{H}(b, x) I_{n+1}(b y)\right\}+\eta(y-x)\left\{\frac{(x / y)^{n+1}}{4 b^{2}(n+1)}-\right.
$$

$$
\left.-2 F_{n 2}^{H}(b, y) I_{n+1}(b x)\right\}+\frac{[1-\eta(H+n)] I_{n+1}\left(\lambda_{n 0} x\right) I_{n+1}\left(\lambda_{n 0} y\right) \bar{A}_{(n) 0}(x, y)}{\lambda_{n 0}^{2}\left(\lambda_{n 0}^{2}-b^{2}\right) I_{n}\left(\lambda_{n 0} x\right) I_{n}\left(\lambda_{n 0} y\right)}
$$

The cases considered above enable us to find further summation formulae for simpler series as well as for more complicated ones. A considerable number of interesting relations can be obtained by allowing $x$ or $y$ to tend to 1 . Such formulae as well as the applications of them are given in [2] and we do not quote them here.

Replacing $a$ or $b$ in equations (3.1)-(3.9) by $i a$ or $i b(i=\sqrt{-1})$, respectively, we can easily obtain another set of summation formulae for the series involving in their denominators the differences of squares. In this case it suffices to use the following relations [1]:

$$
K_{n}(i p)=-\frac{\pi}{2} i^{-n}\left\{Y_{n}(p)+i J_{n}(p)\right\}, \quad I_{n}(i p)=i^{n} J_{n}(p)
$$
obtain

Substituting the right-hand sides of (3.10) into $(2.18)_{2}$ and (2.19) we

$$
F_{n 1}^{H I}(i p, z)=-i^{-n} G_{n 1}^{H}(p, z), \quad F_{n 2}^{H}(i p, z)=i^{-n+1} G_{n 2}^{H}(p, z),
$$

where

$$
\begin{aligned}
& G_{n 1}^{H}(p, z)=\frac{\pi}{8 p^{2}}\left\{J_{n}(p z) \frac{(H+n) Y_{n}(p)-p Y_{n+1}(p)}{(H+n) J_{n}(p)-p J_{n+1}(p)}-Y_{n}(p z)\right\} \\
& G_{n 2}^{H}(p, z)=\frac{\pi}{8 p^{2}}\left\{J_{n+1}(p z) \frac{(H+n) Y_{n}(p)-p Y_{n+1}(p)}{(H+n) J_{n}(p)-p J_{n+1}(p)}-Y_{n+1}(p z)\right\} .
\end{aligned}
$$

Then making use of (3.11) and (3.12) and formulae (2.17) and (3.1)-(3.9) we can derive the following set of summation formulae:

$$
\begin{gathered}
\sum_{j=1}^{\infty} \frac{\lambda_{n j}^{2} J_{n}\left(\lambda_{n j} x\right) J_{n}\left(\lambda_{n j} y\right)}{\left(\lambda_{n j}^{2}-a^{2}\right)\left(\lambda_{n j}^{2}-b^{2}\right)\left(\lambda_{n j}^{2}-n^{2}+H^{2}\right) J_{n}^{2}\left(\lambda_{n j}\right)} \\
=\tilde{A}_{(n) 0}(x, y)+2\left(a^{2}-b^{2}\right)^{-1}\left\{\eta(x-y)\left[a^{2} G_{n 1}^{H}(a, x) J_{n}(a y)-b^{2} G_{n 1}^{H}(b, x) J_{n}(b y)\right]+\right. \\
\left.\quad+\eta(y-x)\left[a^{2} G_{n 1}^{H}(a, y) J_{n}(a x)-b^{2} G_{n 1}^{H}(b, y) J_{n}(b x)\right]\right\},
\end{gathered}
$$


where

$$
\tilde{A}_{(n) 0}(x, y)= \begin{cases}\frac{-A_{(n) 0}(x, y)}{\left(\lambda_{n 0}^{2}+a^{2}\right)\left(\lambda_{n 0}^{2}+b^{2}\right)} & \text { if } H+n \neq 0 \\ -\frac{(n+1) x^{n} y^{n}}{a^{2} b^{2}} & \text { if } H+n=0\end{cases}
$$

$$
\sum_{j=1}^{\infty} \frac{\lambda_{n j}^{2} J_{n}\left(\lambda_{n j} x\right) J_{n}\left(\lambda_{n j} y\right)}{\left(\lambda_{n j}^{2}-a^{2}\right)\left(\lambda_{n j}^{2}+b^{2}\right)\left(\lambda_{n j}^{2}-n^{2}+H^{2}\right)}
$$

$=\tilde{\tilde{A}}_{(n) 0}(x, y)+2\left(a^{2}+b^{2}\right)^{-1}\left\{\eta(x-y)\left[a^{2} G_{n 1}^{H}(a, x) J_{n}(a y)-b^{2} F_{n 1}^{H}(b, x) I_{n}(b y)\right]+\right.$

$$
\left.+\eta(y-x)\left[a^{2} G_{n 1}^{H}(a, y) J_{n}(a x)-b^{2} F_{n 1}^{H}(b, y) I_{n}(b x)\right]\right\} \text {, }
$$

where

$$
\tilde{\tilde{A}}_{(n) 0}(x, y)= \begin{cases}\frac{-A_{(n) 0}(x, y)}{\left(\lambda_{n 0}^{2}+a^{2}\right)\left(\lambda_{n 0}^{2}-b^{2}\right)} & \text { if } H+n \neq 0 \\ \frac{(n+1) x^{n} y^{n}}{a^{2} b^{2}} & \text { if } H+n=0\end{cases}
$$

$$
\begin{aligned}
& \sum_{j=1}^{\infty} \frac{\lambda_{n j}^{3} J_{n+1}\left(\lambda_{n j} x\right) J_{n}\left(\lambda_{n j} y\right)}{\left(\lambda_{n j}^{2}-a^{2}\right)\left(\lambda_{n j}^{2}-b^{2}\right)\left(\lambda_{n j}^{2}-n^{2}+H^{2}\right) J_{n}^{2}\left(\lambda_{n j}\right)} \\
& =2\left(a^{2}-b^{2}\right)^{-1}\left\{\eta(x-y)\left[a^{3} G_{n 2}^{H}(a, x) J_{n}(a y)-b^{3} G_{n 2}^{H}(b, x) J_{n}(b y)\right]+\right. \\
& \left.+\eta(y-x)\left[a^{3} G_{n 1}^{H}(a, y) J_{n+1}(a x)-b^{3} G_{n 1}^{H}(b, y) J_{n+1}(b x)\right]\right\}+ \\
& \quad+\frac{\lambda_{n 0}[1-\eta(H+n)] I_{n+1}\left(\lambda_{n 0} x\right)}{\left(\lambda_{n 0}^{2}+a^{2}\right)\left(\lambda_{n 0}^{2}+b^{2}\right) I_{n}\left(\lambda_{n 0} x\right)} \bar{A}_{(n) 0}(x, y)
\end{aligned}
$$

$$
\begin{gathered}
\sum_{j=1}^{\infty} \frac{\lambda_{n j}^{3} J_{n+1}\left(\lambda_{n j} x\right) J_{n}\left(\lambda_{n j} y\right)}{\left(\lambda_{n j}^{2}-a^{2}\right)\left(\lambda_{n j}^{2}+b^{2}\right)\left(\lambda_{n j}^{2}-n^{2}+H^{2}\right) J_{n}^{2}\left(\lambda_{n j}\right)} \\
=2\left(a^{2}+b^{2}\right)^{-1}\left\{\eta(x-y)\left[a^{3} G_{n 2}^{H}(a, x) J_{n}(a y)-b^{3} F_{n 2}^{H}(b, x) I_{n}(a y)\right]+\right. \\
\left.+\eta(y-x)\left[a^{3} G_{n 1}^{H}(a, y) J_{n+1}(a x)+b^{3} F_{n 1}^{H}(b, y) I_{n+1}(a x)\right]\right\}+ \\
\quad+\frac{\lambda_{n 0}[1-\eta(H+n)] I_{n+1}\left(\lambda_{n 0} x\right)}{\left(\lambda_{n 0}^{2}+a^{2}\right)\left(\lambda_{n 0}^{2}-b^{2}\right) I_{n}\left(\lambda_{n 0} x\right)} \bar{A}_{(n) 0}(x, y),
\end{gathered}
$$

$$
\begin{aligned}
& \sum_{j=1}^{\infty} \frac{\lambda_{n j} J_{n+1}\left(\lambda_{n j} x\right) J_{n}\left(\lambda_{n j} y\right)}{\left(\lambda_{n j}^{2}-a^{2}\right)\left(\lambda_{n j}^{2}-b^{2}\right)\left(\lambda_{n j}^{2}-n^{2}+H^{2}\right) J_{n}^{2}\left(\lambda_{n j}\right)} \\
& =2\left(a^{2}-b^{2}\right)^{-1}\left\{\eta(x-y)\left[a G_{n 2}^{H}(a, x) J_{n}(a y)-b G_{n 2}^{H}(b, x) J_{n}(b y)\right]+\right. \\
& \left.+\eta(y-x)\left[a G_{n 1}^{H}(a, y) J_{n+1}(a x)-b G_{n 1}^{H}(b, y) J_{n+1}(b x)\right]\right\}+ \\
& \quad+\frac{[\eta(H+n)-1] I_{n+1}\left(\lambda_{n 0} x\right) \bar{A}_{(n) 0}(x, y)}{\lambda_{n 0}\left(\lambda_{n 0}^{2}+a^{2}\right)\left(\lambda_{n 0}^{2}+b^{2}\right) I_{n}\left(\lambda_{n 0} x\right)}+\frac{\eta(x-y)}{2 a^{2} b^{2} x}\left(\frac{y}{x}\right)^{n},
\end{aligned}
$$




$$
\begin{aligned}
& \sum_{j=1}^{\infty} \frac{\lambda_{n j} J_{n+1}\left(\lambda_{n j} x\right) J_{n}\left(\lambda_{n j} y\right)}{\left(\lambda_{n j}^{2}-a^{2}\right)\left(\lambda_{n j}^{2}+b^{2}\right)\left(\lambda_{n j}^{2}-n^{2}+H^{2}\right) J_{n j}^{2}\left(\lambda_{n j}\right)} \\
& =2\left(a^{2}+b^{2}\right)^{-1}\left\{\eta(x-y)\left[a G_{n 2}^{H}(a, x) J_{n}(a y)+b^{2} F_{n 2}^{H}(b, x) I_{n}(b y)\right]+\right. \\
& \left.\quad+\eta(y-x)\left[a G_{n 1}^{H}(a, y) J_{n+1}(a x)-b F_{n 1}^{H}(b, y) I_{n+1}(b x)\right]\right\}+ \\
& \quad+\frac{[\eta(H+n)-1] I_{n+1}\left(\lambda_{n 0} x\right) \bar{A}_{(n) 0}(x, y)}{\lambda_{n 0}\left(\lambda_{n 0}^{2}+a^{2}\right)\left(\lambda_{n 0}^{2}-b^{2}\right) I_{n}\left(\lambda_{n 0} x\right)}-\frac{\eta(x-y)}{2 a^{2} b^{2} x}\left(\frac{y}{x}\right)^{n}, \\
& \sum_{j=1}^{\infty} \frac{\lambda_{n j}^{2} J_{n+1}\left(\lambda_{n j} x\right) J_{n+1}\left(\lambda_{n j} y\right)}{\left(\lambda_{n j}^{2}-a^{2}\right)\left(\lambda_{n j}^{2}-b^{2}\right)\left(\lambda_{n j}^{2}-n^{2}+H^{2}\right) J_{n j}^{2}\left(\lambda_{n j}\right)} \\
& =2\left(a^{2}-b^{2}\right)^{-1}\left\{\eta(x-y)\left[a^{2} G_{n 2}^{H}(a, x) J_{n+1}(a y)-b^{2} G_{n 2}^{H}(b, x) J_{n+1}(b y)\right]+\right. \\
& \left.\quad+\eta(y-x)\left[a^{2} G_{n 2}^{H}(a, y) J_{n+1}(a x)-b^{2} G_{n 2}^{H}(b, y) J_{n+1}(b x)\right]\right\}+ \\
& \quad+\frac{[\eta(H+n)-1] I_{n+1}\left(\lambda_{n 0} x\right) I_{n+1}\left(\lambda_{n 0} y\right)}{\left(\lambda_{n 0}^{2}+a^{2}\right)\left(\lambda_{n 0}^{2}+b^{2}\right) I_{n}\left(\lambda_{n 0} x\right) I_{n}\left(\lambda_{n 0} y\right)} \bar{A}_{(n) 0}(x, y),
\end{aligned}
$$

$$
\sum_{j=1}^{\infty} \frac{\lambda_{n j}^{2} J_{n+1}\left(\lambda_{n j} x\right) J_{n+1}\left(\lambda_{n j} y\right)}{\left(\lambda_{n j}^{2}-a^{2}\right)\left(\lambda_{n j}^{2}+b^{2}\right)\left(\lambda_{n j}^{2}-n^{2}+H^{2}\right) J_{n}^{2}\left(\lambda_{n j}\right)}
$$

$$
\begin{aligned}
=2\left(a^{2}+b^{2}\right)^{-1}\left\{\eta(x-y)\left[a^{2} G_{n 2}^{H}(a, x) J_{n+1}(a y)-b^{2} F_{n 2}^{H}(b, x) I_{n+1}(b y)\right]+\right. \\
\left.+\eta(y-x)\left[a^{2} G_{n 2}^{H}(a, y) J_{n+1}(a x)-b^{2} F_{n 2}^{H}(b, y) I_{n+1}(b x)\right]\right\}+ \\
+\frac{[\eta(H+n)-1] I_{n+1}\left(\lambda_{n 0} x\right) I_{n+1}\left(\lambda_{n 0} y\right) \bar{A}_{(n) 0}(x, y)}{\left(\lambda_{n 0}^{2}+a^{2}\right)\left(\lambda_{n 0}^{2}-b^{2}\right) I_{n}\left(\lambda_{n 0} x\right) I_{n}\left(\lambda_{n 0} y\right)} .
\end{aligned}
$$

Further interesting relations can be obtained by allowing $x$ or $y$ to tend to 1. Derivation of the summation formulae for the series involving denominators with more complicated combinations of products of sums of squares and of differences of squares is also very simple. In particular, for a single difference of squares one can immediately obtain the summation formulae substituting $i a$ instead of $a$ into (2.17), (3.1), (3.3) and (3.4).

\section{Sums that can be expressed by combinations of the Kelvin functions.}

Replacing $a$ and $b$ in equations (3.13), (3.14) and others by $a \sqrt{i}$ we obtain the sums of the series that involve the binomial $\lambda_{n j}^{4}+a^{4}$. These sums will be expressed by combinations of the Kelvin functions (see [3] and [8]) in their polar form. Generally, the substitution into any of the relations written above $i a$ and/or $i b$ instead of $a$ and/or $b$ leads to the sums expressable by the Kelvin functions.

Let us make the mentioned substitution in (3.13) and (3.14) for $a=b$. We obtain 


$$
\sum_{j=1}^{\infty} \frac{\lambda_{n j}^{2} J_{n}\left(\lambda_{n j} x\right) J_{n}\left(\lambda_{n j} y\right)}{\left(\lambda_{n j}^{4}+a^{4}\right)\left(\lambda_{n j}^{2}-n^{2}+H^{2}\right) J_{n}^{2}\left(\lambda_{n j}\right)}
$$

$=\hat{A}_{(n) 0}(x, y)+\frac{M_{n}(a x) M_{n}(a y)}{2 a^{2}}\left\{-\left[\eta(x-y) \frac{N_{n}(a x)}{M_{n}(a x)} \sin \left[\varphi_{n}(a x)+\theta_{n}(a y)\right]+\right.\right.$ $\left.+\eta(y-x) \frac{N_{n}(a y)}{M_{n}(a y)} \sin \left[\varphi_{n}(a y)+\theta_{n}(a x)\right]\right]+$

$+[\eta(x-y)+\eta(y-x)]\left((H+n)^{2} N_{n}(a) M_{n}(a) \sin \varphi_{1}(0)+\right.$

$+a^{2} N_{n+1}(a) M_{n+1}(a) \sin \varphi_{2}(0)+a(H+n)\left[N_{n}(a) M_{n+1}(a) \cos \varphi_{3}(0)-\right.$

$\left.\left.-M_{n}(a) N_{n+1}(a) \cos \varphi_{4}(0)\right]\right)\left((H+n)^{2} M_{n}^{2}(a)+a^{2} M_{n+1}^{2}(a)+\right.$

$$
\left.\left.+2 a(H+n) M_{n}(a) M_{n+1}(a) \cos \left[\theta_{n+1}(a)-\theta_{n}(a)-\frac{\pi}{4}\right]\right)^{-1}\right\},
$$

where

$$
\hat{A}_{(n) 0}(x, y)= \begin{cases}\frac{-A_{(n) 0}(x, y)}{\lambda_{n 0}^{4}+a^{4}} & \text { if } \boldsymbol{H}+n \neq 0, \\ -(n+1) x^{n} y^{n} a^{-4} & \text { if } \boldsymbol{H}+n=0 ;\end{cases}
$$

$$
\sum_{j=1}^{\infty} \frac{\lambda_{n j}^{2} J_{n+1}\left(\lambda_{n j} x\right) J_{n+1}\left(\lambda_{n j} y\right)}{\left(\lambda_{n j}^{4}+a^{4}\right)\left(\lambda_{n j}^{2}-n^{2}+H^{2}\right) J_{n}^{2}\left(\lambda_{n j}\right)}
$$

$$
\begin{aligned}
= & \frac{[\eta(H+n)-1] I_{n+1}\left(\lambda_{n 0} x\right) I_{n+1}\left(\lambda_{n 0} y\right)}{\left(\lambda_{n 0}^{4}+a^{4}\right) I_{n}\left(\lambda_{n 0} x\right) I_{n}\left(\lambda_{n 0} y\right)} \bar{A}_{(n) 0}+ \\
& +\frac{M_{n+1}(a x) M_{n+1}(a y)}{2 a^{2}}\left\{-\left[\eta(x-y) \frac{N_{n+1}(a x)}{M_{n+1}(a x)} \sin \left[\varphi_{n+1}(a x)+\theta_{n+1}(a y)\right]+\right.\right.
\end{aligned}
$$$$
\left.+\eta(y-x) \frac{N_{n+1}(a y)}{M_{n+1}(a y)} \sin \left[\varphi_{n+1}(a y)+\theta_{n+1}(a x)\right]\right]+
$$$$
+[\eta(x-y)+\eta(y-x)]\left((H+n)^{2} N_{n}(a) M_{n}(a) \sin \varphi_{1}(1)+\right.
$$$$
+a^{2} N_{n+1}(a) M_{n+1}(a) \sin \varphi_{2}(1)+a(H+n)\left[N_{n}(a) M_{n+1}(a) \cos \varphi_{3}(1)-\right.
$$$$
\left.\left.-M_{n}(a) N_{n+1}(a) \cos \varphi_{4}(1)\right]\right)\left((H+n)^{2} M_{n}^{2}(a)+a^{2} M_{n+1}^{2}(a)+\right.
$$$$
\left.\left.+2 a(H+n) M_{n}(a) M_{n+1}(a) \cos \left[\theta_{n+1}(a)-\theta_{n}(a)-\frac{\pi}{4}\right]\right)^{-1}\right\},
$$

where

$$
\begin{aligned}
& \varphi_{1}(k)=\theta_{n+k}(a x)+\theta_{n+k}(a y)+\varphi_{n}(a)-\theta_{n}(a), \\
& \varphi_{2}(k)=\theta_{n+k}(a x)+\theta_{n+k}(a y)+\varphi_{n+1}(a)-\theta_{n+1}(a), \\
& \varphi_{3}(k)=\theta_{n+k}(a x)+\theta_{n+k}(a y)+\varphi_{n}(a)-\theta_{n+1}(a)-\pi / 4, \\
& \varphi_{4}(k)=\theta_{n+k}(a x)+\theta_{n+k}(a y)+\varphi_{n+1}(a)-\theta_{n}(a)+\pi / 4,
\end{aligned}
$$




$$
\begin{array}{cl}
M_{n}(z)=\sqrt{\operatorname{ber}_{n}^{2} z+\operatorname{bei}_{n}^{2} z}, & N_{n}(z)=\sqrt{\operatorname{ker}_{n}^{2} z+\operatorname{kei}_{n}^{2} z} \\
\theta_{n}(z)=\operatorname{arctg}\left(\operatorname{bei}_{n} z / \operatorname{ber}_{n} z\right), & \varphi_{n}(z)=\operatorname{arctg}\left(\operatorname{kei}_{n} z / \operatorname{ker}_{n} z\right)
\end{array}
$$

$\operatorname{ber}_{n} z, \operatorname{bei}_{n} z, \operatorname{ker}_{n} z, \operatorname{kei}_{n} z$ are the Kelvin functions of order $n$ (see [3] and [8]). They are related to the Bessel functions $J_{n}(z), Y_{n}(z), I_{n}(z)$ and $K_{n}(z)$ by the following formulae:

$$
\begin{gathered}
J_{n}(\sqrt{i z})=(-1)^{n} M_{n}(z) \exp \left[-i \theta_{n}(z)\right]=(-1)^{n}\left(\operatorname{ber}_{n} z-i \operatorname{bei}_{n} z\right) \\
I_{n}(\sqrt{i} z)=(-i)^{n} M_{n}(z) \exp \left[i \theta_{n}(z)\right]=(-i)^{n}\left(\operatorname{ber}_{n} z+i \operatorname{bei}_{n} z\right) \\
K_{n}(\sqrt{i z})=i^{n} N_{n}(z) \exp \left[i \varphi_{n}(z)\right]=i^{n}\left(\operatorname{ker}_{n} z+i \operatorname{kei}_{n} z\right) \\
Y_{n}(\sqrt{i} z)=(-1)^{n}\left\{i M_{n}(z) \exp \left[-i \theta_{n}(z)\right]-\frac{2}{\pi} N_{n}(z) \exp \left[-i \varphi_{n}(z)\right]\right\} \\
=(-1)^{n}\left\{\operatorname{bei}_{n} z+i \operatorname{ber}_{n} z-\frac{2}{\pi}\left(\operatorname{ker}_{n} z-i \operatorname{kei}_{n} z\right)\right\}
\end{gathered}
$$

The formulae derived above seem to be rather complicated. We could obtain further summation formulae like (4.1) or (4.2). However, the most important point seems to be the possibility of performing such a summation.

5. Conclusions. In many cases the solution of the differential equations takes the form of a Dini series. The summation formulae given above make the physical interpretation of such a solution possible. Application of the summation formulae for the Dini series as well as some particular cases interesting from the point of view of the theory of continuous media are shown in [2]. It is also obvious that summation of the series mentioned in the present paper liberates us from the tiring procedure of determining the values of the roots of transcendental equations. That seems to be one of the most essential advantages of the formulae worked out in this paper.

Acknowledgement. The authors wish to express their gratitude to Dr. D. E. Taylor from the University of Sydney for helpful comments on the use of the English language.

\section{Referonces}

[1] K. Grysa, O sumowaniu pewnych szeregbro Fouriera-Bessela, Mech. Teoret. Stos. 15 (1977), p. 205-214.

[2] - and J. Jankowski, $O$ sumowaniu pewnych szeregów Diniogo i trygonometrycznyoh pojaroiajqoych się w zagadnieniach meohanilki ofrodkóno ciaglych, ibidem 16 (1978), p. 299-319.

[3] N. W. MeLachlan, Funkeje Bessela dla inżynierón, Warbzawa 1964.

9 - Zastosowania Matematyki $\mathbf{1 7 . 1}$ 
[4] J. Mikusiński and R. Sikorski, Elementarna teoria dystrybucji, Warszawa 1964.

[5] I. N. Sned don, Fourier transforms, McGraw-Hill Book Company Inc., 1951.

[6] - Mixed boundary value problems in potential theory, Amsterdam 1966.

[7] Z. Szmydt, Fourier transformation and linear differential equations, Dordrecht 1977.

[8] G. N. Watson, A treatise on the theory of Bessel functions, Cambridge 1962.

[9] S. Woelke, Summation of certain Bessel series occurring in elasticity problems, Arch. Mech. Stos. 22 (1970), p. 303 - 314.

INSTITUTE OF TECHNICAL MECHANICS

TEOHNICAL UNIVERSITY POZNAN

Received on 23. 3. 1977;

revised version on 8.6 .1978

K. GRYSA i J. JANKO WSKI (Poxná́)

\section{O SUMO WANIU PEWNYCH SZEREGóW DINIEGO}

\section{STRESZCZENIE}

W pracy wyprowadzono wzory na sumy pewnych szeregów Diniego, zależnych od dwu zmiennych. Sumowalność tych szeregów uzasadniono na gruncie teorii dystrybucji, korzystając z własności ciągów deltowych. Działania dystrybucyjne, wykonywane $w$ pracy na tych szeregach, prowadza do wyników będących dystrybucjami regularnymi. Pozwala to wysumować pewne szeregi, pojawiające się np. w problemach mechaniki ośrodk6w ciagłych. 